\title{
Awareness of ovarian cancer symptoms in general population
}

\author{
M. Bishtawi ${ }^{1}$, H. Saleh ${ }^{2}$ \\ ${ }^{1}$ Al Ahli Hospital, Obstetrics and Gynecology, Doha, Qatar. \\ 2Sidra medicine, Obstetrics and Gynecology, Doha, Qatar.
}

\section{Objectives:}

Ovarian cancer accounts for $3 \%$ of all female cancers and has a high mortality rate among gynecological malignancies. Early diagnosis carries a high survival rate of $93 \%$. So, this study was carried out to assess the knowledge and awareness of Jordanian women about ovarian cancer symptoms and risk factors.

\section{Methods:}

A cross-sectional survey design was used. women randomly selected to complete the survey, 896 women completed the survey

\section{Results}

The mean of total symptoms recognized was low at level of $3.2(\mathrm{SD}=2.7$ ) out of 10 . The three highest known symptoms among women were as follows: extreme fatigue (43.2\%), back pain (42.4\%), and persistent pain in pelvic area $(40.7 \%)$. The most commonly known risk factor was smoking $(68.4 \%)$, followed by having ovarian cyst(s) $(59.7 \%)$

\section{Conclusions:}

Poor awareness of ovarian cancer risk factors and symptoms were noticed. awareness need to be raised through education and social media. The absence of an effective screening program, a national awareness campaign is urgently needed to improve the public's understanding of symptoms and risk factors and increasing women's confidence in symptom recognition
Table 2. Frequency and Percentage of Women Knowledge of Ovarian Cancer Symptoms.

\begin{tabular}{|c|c|c|c|c|c|}
\hline \multirow{2}{*}{\multicolumn{2}{|c|}{ Symptom }} & \multicolumn{2}{|l|}{ Yes } & \multicolumn{2}{|l|}{ No } \\
\hline & & \multirow{2}{*}{$\begin{array}{c}\text { Frequency } \\
334\end{array}$} & \multirow{2}{*}{$\begin{array}{c}\% \\
37.3\end{array}$} & \multirow{2}{*}{$\begin{array}{c}\text { Frequency } \\
562\end{array}$} & \multirow{2}{*}{$\begin{array}{c}\% \\
62.7\end{array}$} \\
\hline I. & Persistent pain in your abdomen & & & & \\
\hline 2. & Persistent pain in your pelvis & 365 & 40.7 & 531 & 59.3 \\
\hline 3. & Persistent bloating & 250 & 27.9 & 646 & 72.15 \\
\hline 4. & $\begin{array}{l}\text { Increased abdominal size on } \\
\text { most days }\end{array}$ & 337 & 37.6 & 559 & 62.4 \\
\hline 5. & Feeling full persistently & 132 & 14.7 & 764 & 85.3 \\
\hline 6. & Difficulty eating on most days & 114 & 12.7 & 782 & 87.3 \\
\hline 7. & Passing more urine than usual & 267 & 29.8 & 629 & 70.2 \\
\hline 8. & Changes in bowel habit & 282 & 31.5 & 614 & 68.5 \\
\hline 9. & Extreme fatigue & 387 & 43.2 & 509 & 56.8 \\
\hline 10. & Back pain & 380 & 42.4 & 516 & 57.6 \\
\hline
\end{tabular}

Table 3. Frequency and Percentage of Women Knowledge of Ovarian Cancer Risk Factors.

\begin{tabular}{|c|c|c|c|c|c|}
\hline \multirow{2}{*}{\multicolumn{2}{|c|}{ Risk factor }} & \multicolumn{2}{|l|}{ Yes } & \multicolumn{2}{|l|}{ No } \\
\hline & & \multirow{2}{*}{$\begin{array}{c}\text { Frequency } \\
380\end{array}$} & \multirow{2}{*}{$\begin{array}{c}\% \\
42.4\end{array}$} & \multirow{2}{*}{$\begin{array}{c}\text { Frequency } \\
516\end{array}$} & \multirow{2}{*}{$\begin{array}{c}\% \\
57.6\end{array}$} \\
\hline I. & $\begin{array}{l}\text { Having a close relative with } \\
\text { ovarian cancer }\end{array}$ & & & & \\
\hline 2. & $\begin{array}{l}\text { Having a past history of breast } \\
\text { cancer }\end{array}$ & 418 & 46.7 & 478 & 53.3 \\
\hline 3. & Using HRT & 401 & 44.8 & 495 & 55.2 \\
\hline 4. & Being overweight (BMI over 25) & 345 & 38.5 & 551 & 61.5 \\
\hline 5. & Having endometriosis & 341 & 38.1 & 555 & 61.9 \\
\hline 6. & Having ovarian cysts & 535 & 59.7 & 361 & 40.3 \\
\hline 7. & $\begin{array}{l}\text { Using talcum powder in the } \\
\text { genital area }\end{array}$ & 254 & 28.3 & 642 & 71.7 \\
\hline 8. & Being over 50 years old & 370 & 41.3 & 526 & 58.7 \\
\hline 9. & Having IVF treatment & 255 & 28.5 & 641 & 71.5 \\
\hline 10. & Not having children & 302 & 33.7 & 594 & 66.3 \\
\hline II. & $\begin{array}{l}\text { Having gone through the } \\
\text { menopause }\end{array}$ & 411 & 45.9 & 485 & 54.1 \\
\hline 12. & Being a smoker & 613 & 68.4 & 283 & 31.6 \\
\hline
\end{tabular}

Note. $\mathrm{HRT}=$ hormone replacement therapy; $\mathrm{BMI}=$ body mass index; $\mathrm{IVF}=$ in vitro fertilization.
Table 1. Sample Demographics.

\begin{tabular}{|c|c|c|}
\hline Variable & Frequency $(\%)$ & $M(S D)$ \\
\hline \multirow{2}{*}{\multicolumn{3}{|c|}{ Marital status }} \\
\hline & & \\
\hline Single & $181(20.2)$ & \\
\hline Married & $618(69.0)$ & \\
\hline Divorce or widow & $89(9.9)$ & \\
\hline Not mentioned & $8(0.9)$ & \\
\hline \multicolumn{3}{|l|}{ Education } \\
\hline Low education (secondary school and below) & $399(44.5)$ & \\
\hline High education (diploma and above) & $497(55.5)$ & \\
\hline \multicolumn{3}{|l|}{ Family income } \\
\hline$<1000 \mathrm{JD}$ & $751(83.8)$ & \\
\hline$\geq 1000 \mathrm{JD}$ & $145(16.2)$ & \\
\hline \multicolumn{3}{|l|}{ Work status } \\
\hline Full-time Job & $705(78.7)$ & \\
\hline Not working or retired & $191(21.3)$ & \\
\hline \multicolumn{3}{|l|}{ Knowledge of person with cancer } \\
\hline Yes & $47(5.3)$ & \\
\hline No & $849(94.7)$ & \\
\hline \multicolumn{3}{|l|}{ Living region } \\
\hline Central & $475(53.1)$ & \\
\hline Northern & $270(30.1)$ & \\
\hline Southern & 208 (16.8) & \\
\hline
\end{tabular}

Note. JD = Jordanian Dinar.

$208(16.8)$

\begin{tabular}{|c|c|c|c|c|}
\hline Variable & $M(S D)$ & $t$ & $p$ value & $\begin{array}{l}95 \% \mathrm{Cl} \text { of the } \\
\text { difference }\end{array}$ \\
\hline Education level & & -3.96 & $<.001^{*}$ & {$[-1.69,-0.36]$} \\
\hline Low educated & $4.8(2.7)$ & & & \\
\hline $\begin{array}{l}\text { Highly educated } \\
\text { nith }\end{array}$ & $5.5(2.8)$ & & & \\
\hline $\begin{array}{l}\text { Knowing women with ovarian } \\
\text { cancer }\end{array}$ & & 0.31 & 0.756 & {$[-1.15,1.58]$} \\
\hline Yes & $\begin{array}{l}5.4(2.7) \\
5.2(2.8)\end{array}$ & & & \\
\hline $\begin{array}{l}\text { No } \\
\text { Waiting time to seek medical }\end{array}$ & $5.2(2.8)$ & -0.04 & 0.966 & {$[-0.45,0.44]$} \\
\hline $\begin{array}{l}\text { care } \\
\leq 2 \text { weeks }\end{array}$ & $5.2(2.8)$ & & & \\
\hline$>2$ weeks & $5.2(2.6)$ & & & \\
\hline Family monthly income & & -2.84 & $0.005 *$ & {$[-1.20,-0.22]$} \\
\hline Low $(\leq 1000$ JD) & $5.0(2.7)$ & & & \\
\hline $\begin{array}{l}\text { High ( }>1000 \mathrm{JD} \\
\text { Aware of the presence of }\end{array}$ & $5.8(2.9)$ & 4.60 & $<.001 *$ & {$[0.69,1.73]$} \\
\hline $\begin{array}{l}\text { the national breast cancer } \\
\text { screening program }\end{array}$ & & & & \\
\hline Yes & $5.3(2.7)$ & & & \\
\hline No & $4.1(2.8)$ & & & \\
\hline
\end{tabular}

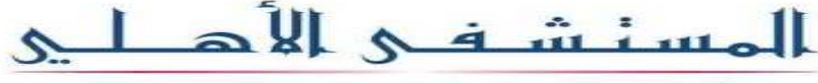
AL-AHLI HOSPITAL 